\title{
Comparison of Mamdani-Type and Sugeno-Type Fuzzy Inference Systems for Transformer Tap Changing System
}

\author{
Charles R. Sarimuthu, Vigna K. Ramachandaramurthy, H. Mokhlis, K. R. Agileswari \\ Department of Electrical Power Engineering, Universiti Tenaga Nasional, Malaysia
}

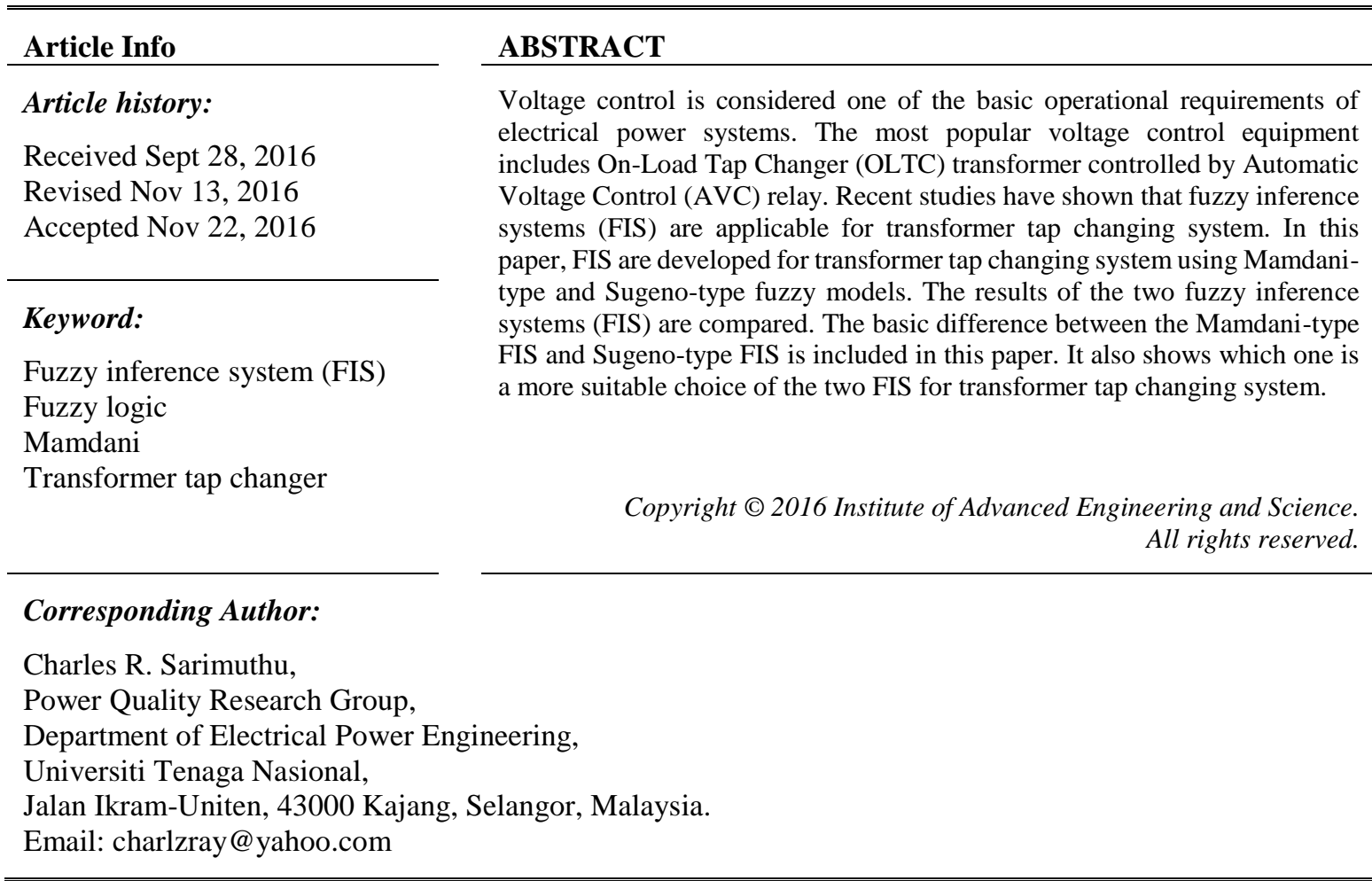

\section{INTRODUCTION}

Fuzzy logic is considered a widely used technology since the late 1980s. Fuzzy logic system is applied successfully in many applications such as consumer products, industrial process control and automotive industries [1]. Fuzzy logic is closely linked to human thinking and natural language than conventional logical systems [2]. In classical control theory, fuzzy control is used to build a model of human expert who is capable of controlling the plant without thinking in terms of mathematical model [3]. Fuzzy systems are very useful in situations involving highly complex systems whose behaviors are not well understood and in situations where an approximate, but fast, solution is warranted [4].

L.A. Zadeh introduced fuzzy logic in 1965. Fuzzy logic application in the subway system in Sendai city of Japan has shown superior results compared to traditional control. The superior result was achieved in subway system of Sendai due to engineers spending several months to obtain correct fuzzy input sets. Similarly, studies have shown that fuzzy systems are applicable in transformer tap changing systems. In 2015, S.K. Salman et al. presented a design of fuzzy logic- based AVC relay for voltage control of distribution network [5]. Fuzzy logic systems are used in the design due to its simple and fast operation. Fuzzy logic system reduces the design development cycle, simplify design complexity, improve control performance, simplify implementation and reduce the hardware costs. In addition, fuzzy rules are more expressive than crisp values [6]. This paper is organized as follows: Section 2 gives the difference between Mamdani-type and Sugeno-type FIS. Section 2.1, shows the development of Mamdani-type FIS. Section 2.2, shows the development of Sugenotype FIS. Section 3, gives results and discussions and section 4 conclusions. 


\section{RESEARCH METHOD}

Mamdani method is well known for capturing expert knowledge and it allows us to describe the expertise in more intuitive, more human-like manner. However, Mamdani-type FIS is not considered to be computationally efficient. On the other hand, Sugeno method is computationally efficient and works well with optimization and adaptive techniques, which makes it very attractive in dynamic non-linear systems. These adaptive techniques can be used to customize the membership functions so that fuzzy system best models the data. The major difference between Mamdani-type FIS and Sugeno-type FIS is the way the crisp output is generated from the fuzzy inputs. While Mamdani-type FIS uses the technique of defuzzification of a fuzzy output, Sugeno-type FIS uses weighted average to compute the crisp output. The expressive power and interpretability of Mamdani output is not found in the Sugeno FIS since the consequents of the rules are not fuzzy [7]. But Sugeno method has better processing time since the weighted average replace the time consuming defuzzification process. Mamdani-type FIS is widely used in particular for decision support application due to the interpretable and intuitive nature of the rule base. Apart from that, Mamdani FIS has output membership functions whereas Sugeno FIS has no output membership functions. Mamdani FIS is known to be less flexible in system design in comparison to Sugeno FIS as latter can be integrated with ANFIS tool to optimize the outputs.

\subsection{Development of Mamdani-Type FIS}

Transformer tap changing system is first modelled using Mamdani fuzzy model. It consists of two inputs from voltage error as shown in Figure 1 and AVC relay feedback voltage as shown in Figure 2 showing the current tap position at the on-load tap changer transformer. The system has one output that controls the voltage by determining the tap position. The voltage error is taken in ranges of $-450 \mathrm{~V}$ to $+450 \mathrm{~V}$. The feedback voltage from the AVC relay is taken in ranges of $0.95002 \mathrm{~V}$ per unit to $1.04998 \mathrm{~V}$ per unit. The output i.e. tap position is taken in $0.93336 \mathrm{~V}$ per unit to $1.06664 \mathrm{~V}$ per unit and have nine triangular membership functions as shown in Figure 3. The rules included in for the transformer tap changing system are described in Table 1.

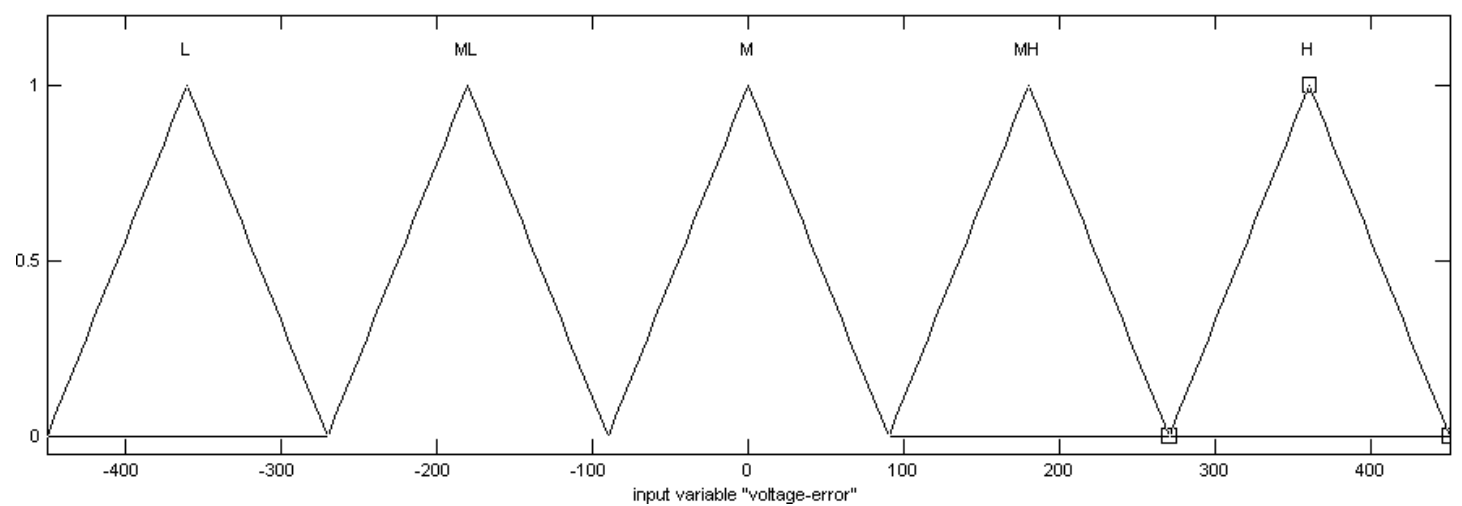

Figure 1. Voltage Error Membership Function

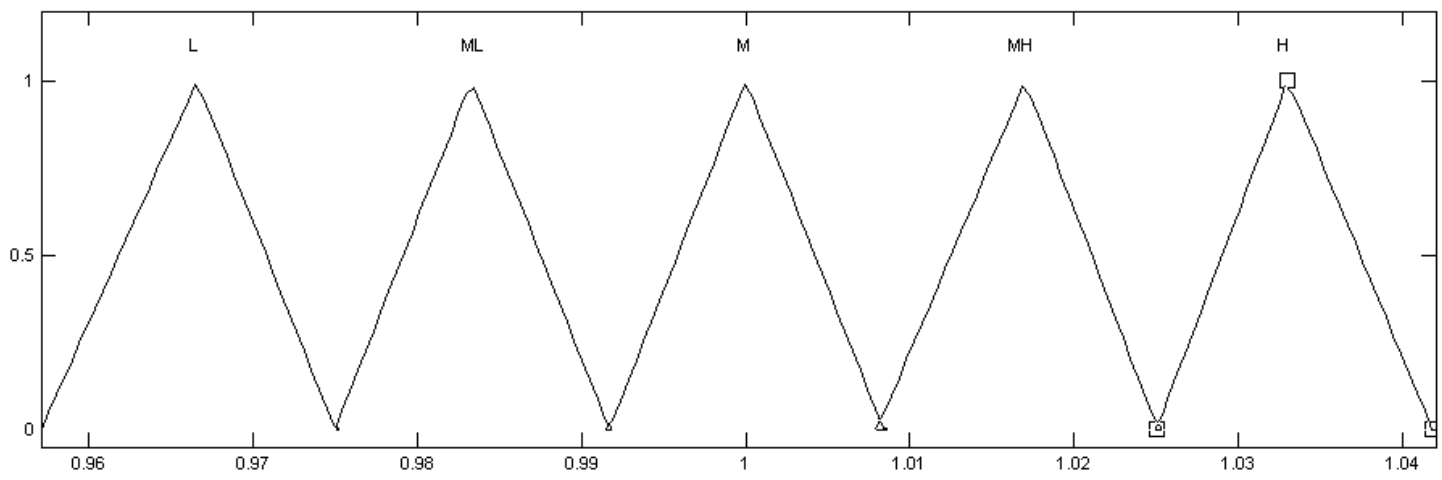

Figure 2. AVC Relay Voltage Feedback from Output Membership Function 


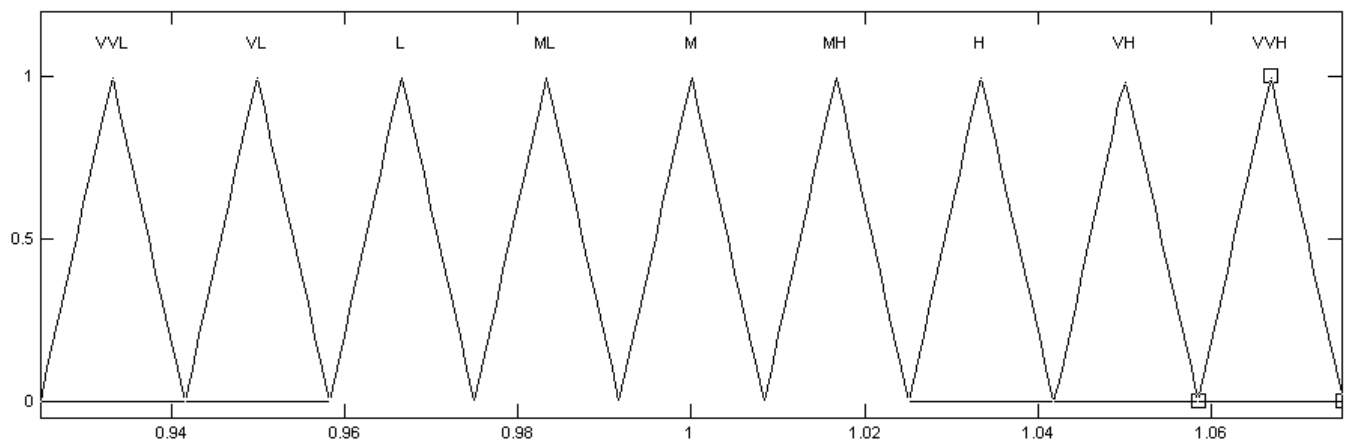

Figure 3. AVC Relay Voltage Membership Functions

Table 1. Rule Base of Mamdani-Type FIS

\begin{tabular}{|c|c|c|c|}
\hline Rules & Voltage error & AVC relay voltage feedback from output & AVC relay voltage \\
\hline 1. & Low & Low & Medium \\
\hline 2. & Low & Medium Low & Medium High \\
\hline 3. & Low & Medium & High \\
\hline 4. & Low & Medium High & Very High \\
\hline 5. & Low & High & Very Very High \\
\hline 6. & Medium Low & Low & Medium Low \\
\hline 7. & Medium Low & Medium Low & Medium \\
\hline 8. & Medium Low & Medium & Medium High \\
\hline 9. & Medium Low & Medium High & High \\
\hline 10. & Medium Low & High & Very High \\
\hline 11. & Medium & Low & Low \\
\hline 12. & Medium & Medium Low & Medium Low \\
\hline 13. & Medium & Medium & Medium \\
\hline 14. & Medium & Medium High & Medium High \\
\hline 15. & Medium & High & High \\
\hline 16. & Medium High & Low & Very Low \\
\hline 17. & Medium High & Medium Low & Low \\
\hline 18. & Medium High & Medium & Medium Low \\
\hline 19. & Medium High & Medium High & Medium \\
\hline 20. & Medium High & High & Medium High \\
\hline 21. & High & Low & Very Very Low \\
\hline 22. & High & Medium Low & Very Low \\
\hline 23. & High & Medium & Low \\
\hline 24. & High & Medium High & Medium Low \\
\hline 25. & High & High & Medium \\
\hline
\end{tabular}

\subsection{Development of Sugeno-Type FIS}

For development of the transformer tap changing system using Sugeno-type model, the initial steps are the same as Mamdani-type model. It also takes input from voltage error and AVC relay feedback voltage and produces a suitable tap position for controlling the voltage at the point of control in the distribution network. Inputs voltage error and feedback voltage from AVC relay have 5 membership function over the ranges of $-450 \mathrm{~V}$ to $+450 \mathrm{~V}$ and $0.95002 \mathrm{~V}$ per unit to $1.04998 \mathrm{~V}$ per unit respectively as already shown in Figure 1 and Figure 2. The output tap position can only be either constant or linear in this FIS, so two membership functions for the output are "no change" and "change" which are constant and are shown in Table 2. The output in Sugeno-type FIS can only be in range of 0-1. The rule base for Sugeno-type FIS is same as for Mamdani-type FIS as shown in Table 1.

Table 2. Tap Position Membership Functions

\begin{tabular}{cc}
\hline Tap position & Constant value \\
\hline No change & 0 \\
Change & 1 \\
\hline
\end{tabular}

\section{RESULTS AND ANALYSIS}

The plots obtained after simulating Mamdani-type of FIS for transformer tap changing system are shown in Figure 4 and Figure 5. 


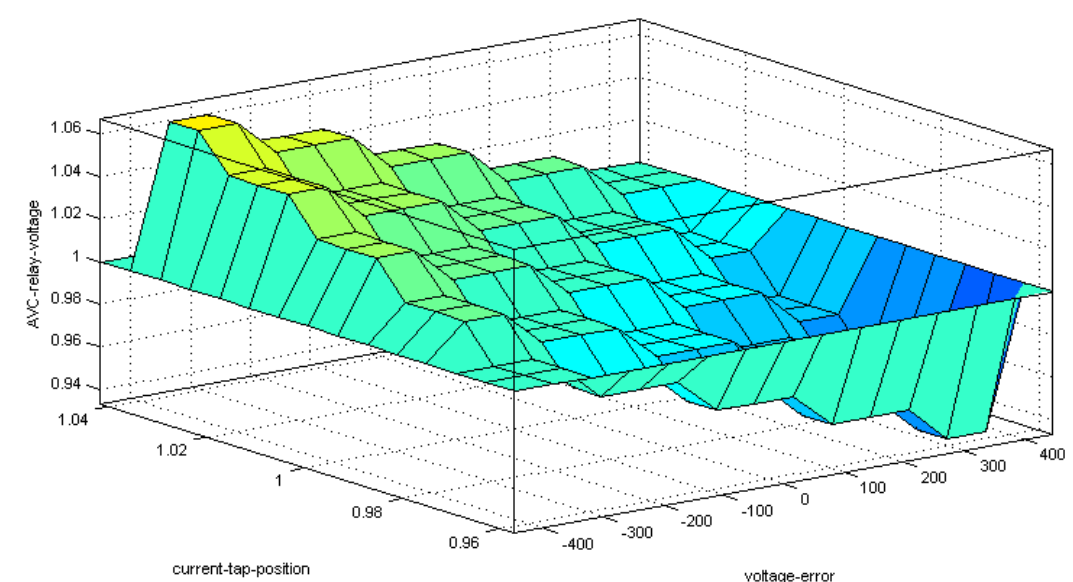

Figure 4. Surface View of Mamdani-Type FIS

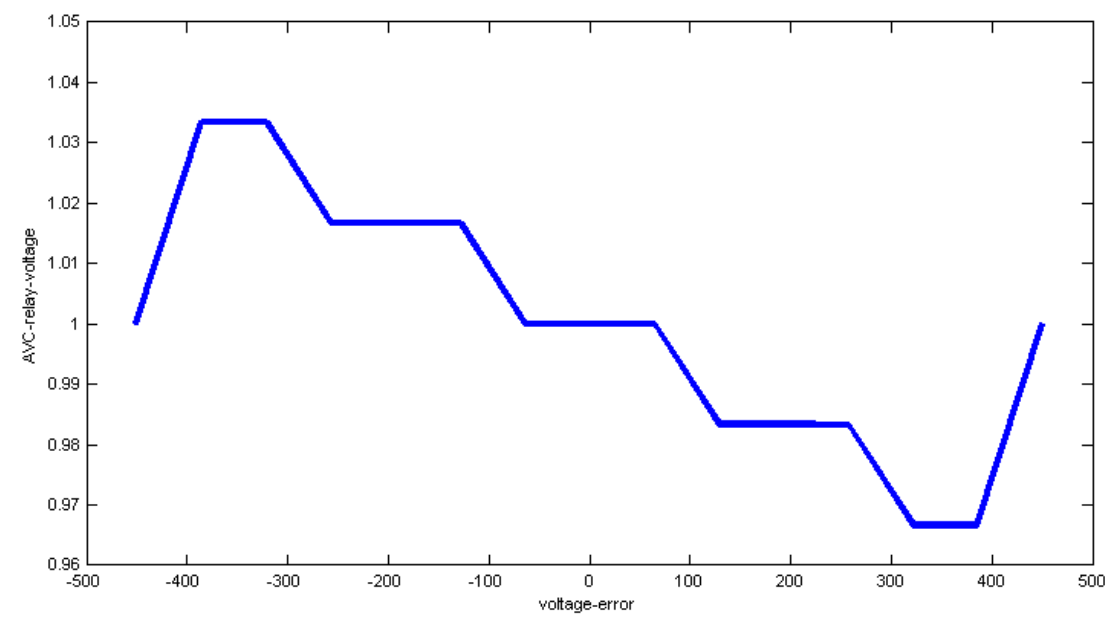

Figure 5. AVC Relay Voltage with Voltage Error Using Mamdani-Type FIS

The following are the plots obtained after simulating the Sugeno-type FIS for transformer tap changing system as shown in Figure 6 and Figure 7.

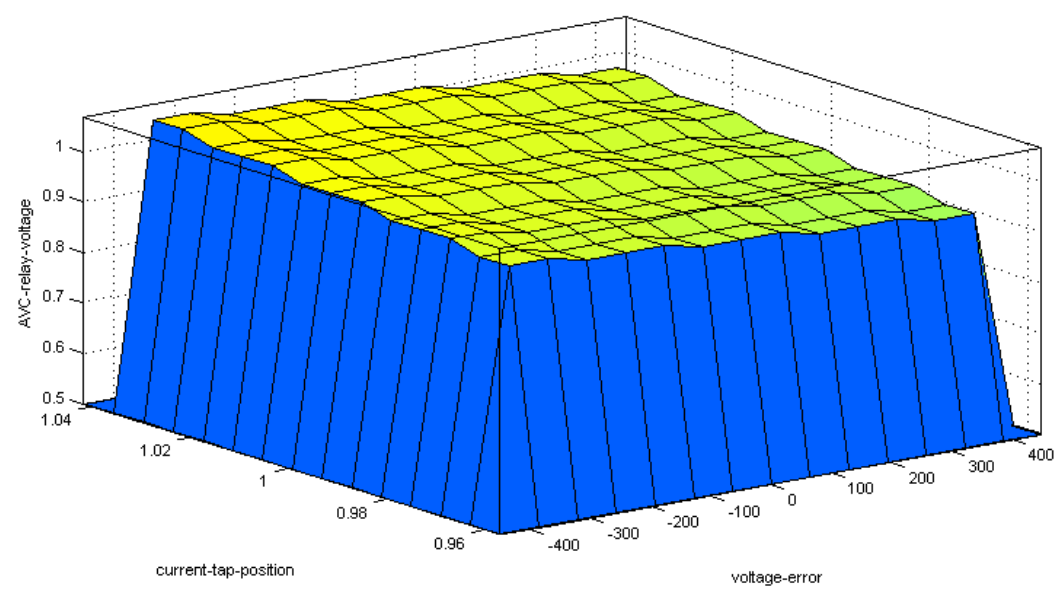

Figure 6. Surface View of Sugeno-Type FIS 


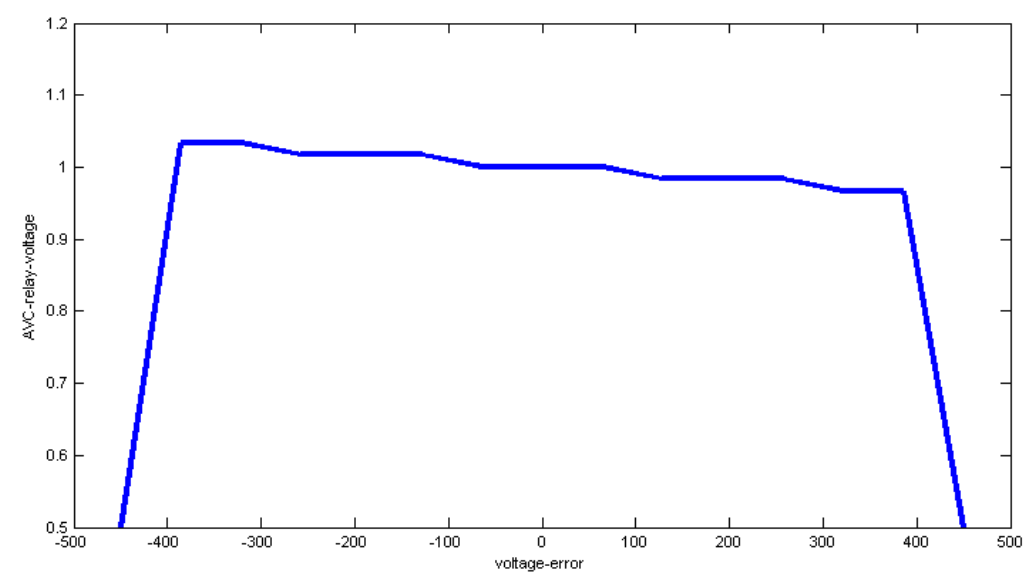

Figure 7. AVC Relay Voltage with Voltage Error Using Sugeno-Type FIS

The results obtained show that for the given application of transformer tap changing system Mamdanitype FIS is more suitable than the Sugeno-type FIS. It is noticed that in cases where the input voltage error goes beyond the range allocated, Sugeno-type FIS transformer tap changing system does not work satisfactorily compared to Mamdani-type FIS. Figure 7 shows that Mamdani-type FIS does not initiate a tap change for extreme conditions where the input voltage error is at levels below or above the described ranges.

\section{CONCLUSION}

It can be concluded from this paper that for transformer tap changing system Mamdani-type FIS performs better than Sugeno-type FIS since it allows the tap to operate at extreme conditions. Although the designing of both the FIS is same but the output membership functions of Sugeno-type can only be either constant or linear and also the crisp output is generated in different ways for both the FIS. Due to this Sugenotype FIS has a disadvantage compared to Mamdani-type FIS in tap changing operation where the latter is able to use defuzzification process to provide interpretation for the most suitable tap position for voltage control.

\section{ACKNOWLEDGEMENT}

This work is supported by Universiti Tenaga Nasional (UNITEN), Taylor's University and University of Malaya, Malaysia.

\section{REFERENCES}

[1] J. Yen and R. Langari, Fuzzy Logic, Pearson Education, 2004.

[2] K. P. Mohandas and S. Karimulla, "Fuzzy and Neuro-Fuzzy Modeling and Control of Non-Linear Systems," Second International Conference on Electrical and Electronics, 2001.

[3] G. S. Sandhu and K. S. Rattan, "Design of a Neuro-Fuzzy Controller," IEEE International Conference on Systems, Man and Cybernetics. 1997.

[4] T. J. Ross, Fuzzy Logic with Engineering Applications, John Wiley and sons, 2010.

[5] Salman SK. and Wan ZG, "Fuzzy Logic-Based AVC Relay for Voltage Control of Distribution Network with and without Distributed/Embedded Generation," IEEE Power Tech Conference, 2007.

[6] Omar A. S. Youssef, "Application of Fuzzy Inference Mechanisms to Power System Relaying," IEEE PES Power System Conference \& Exposition, vol. 1, pp. 560-567, 2004.

[7] A. Haman, and N. D. Geogranas, "Comparison of Mamdani and Sugeno Fuzzy Inference Systems for Evaluating the Quality of Experience of Hapto-Audio-Visual Application," IEEE International Workshop on Haptic Audio Visual Environments and their Applications, 2008. 